\title{
Photoacoustic Tomography Shows the Branching Pattern of Anterolateral Thigh Perforators In Vivo
}

\section{$\operatorname{AUTHOR}(\mathrm{S})$ :}

Tsuge, Itaru; Saito, Susumu; Sekiguchi, Hiroyuki; Yoshikawa, Aya; Matsumoto, Yoshiaki; Toi, Masakazu; Suzuki, Shigehiko

\section{CITATION:}

Tsuge, Itaru ... [et al]. Photoacoustic Tomography Shows the Branching Pattern of Anterolateral Thigh Perforators In Vivo. Plastic and Reconstructive Surgery 2018, 141(5): 1288-1292

\section{ISSUE DATE:}

2018-05

URL:

http://hdl.handle.net/2433/232633

\section{RIGHT:}

(c) 2018American Society of Plastic Surgeons.: This is a non-final version of an article published in final form in Plastic and Reconstructive Surgery, 141(5):1288-1292, May 2018; ; The full-text file will be made open to the public on 01 May 2019 in accordance with publisher's 'Terms and Conditions for Self-Archiving'.; This is not the published version. Please cite only the published version.; この論文は出版社版でありません。引用の際には出版社版をご確認ご利用ください。 


\section{Background}

6 The distal branching pattern of perforators is associated with thin anterolateral thigh (ALT) flap failure. The

7 purpose of this study is to investigate the feasibility of using photoacoustic tomography (PAT) as a diagnostic imaging modality to identify ALT perforators and their branching patterns in the subcutaneous layer.

\section{Methods}

Ten thighs in five healthy males were studied. The anterolateral aspect of the mid-thigh was examined using

11 PAT. The correlation between PAT and ultrasound (US) findings was evaluated. To determine the detectability of PAT by depth, the depth of vessels in the stem portion was compared to the depth of the deep fascia measured by US. Branching patterns of vessels in the adipose and suprafascial layers were evaluated by three-dimensional observation.

\section{Results}

16 A total of 18 perforators were visualized by PAT. PAT and US had comparable diagnostic potential for the

17 detection of perforators. PAT visualized microvessels in the subcutaneous layer, especially those in oblique 
$19 \mathrm{~mm}$. There was a strong correlation between the depth of visualized vessels in the stem portion and the

20 depth of the deep fascia. Three-dimensional observation of PAT images showed the branching morphology

21 of perforators.

\section{Conclusions}

23 This study showed the applicability of PAT to identification of the branching patterns of ALT perforators in

24 vivo although limited visualization of subfascial vessels is a technical issue. We believe PAT has the potential

25 to be a new imaging modality for thin ALT flap surgery.

Anterolateral thigh, imaging, perforator, photoacoustic tomography, thin flap 
Flap thinning ${ }^{1}$ is technically challenging. In particular, a high rate of failure has been reported for the thin anterolateral thigh (ALT) flap. ${ }^{2}$ Previous experimental studies have suggested that skin perfusion is changed during thinning as a result of injuries to vessels in the suprafascial layer. ${ }^{3}$ Thus, an appreciation of the individualized vascular anatomy of the thigh is essential for thin flap planning; however, suitable imaging methods that can be used safely and easily in vivo are lacking. Photoacoustic tomography (PAT) is an emerging vascular imaging modality that has been used in basic research and clinical practice, which allows for visualization of subcutaneous vessels on the basis of the photoacoustic mechanism. Energy from a nearinfrared pulse laser is introduced into hemoglobin. As a result, red blood cells become swollen and release ultrasonic waves, which allows vessels to be visualized without contrast agents. ${ }^{4}$ The purpose of this study is to investigate the feasibility of using PAT as an imaging tool for identifying branching vessels in the subcutaneous layer associated with the thin ALT flap.

\section{Materials and methods}

44 After approval by our institutional ethics committee and clinical trial registration, we recruited five healthy male adults (mean age, 41 years; mean body mass index, $23.1 \mathrm{~kg} / \mathrm{m}^{2}$ ) for this study. PAT was performed bilaterally in all subjects. 
angiogenesis. In that study, the estimated maximum depth of vessels visualized was $27 \mathrm{~mm} .{ }^{5}$ The system ultrasonic transducers were placed into a hemispherical cup built into the platform. Each subject was placed on the platform in the semi-prone position with the anterolateral surface of the mid-thigh facing the cup. The space between the skin and the cup was filled with de-aired water for smooth transmission of ultrasonic waves. Each scan took approximately 2 minutes for a $14 \times 14 \mathrm{~cm}^{2}$ region. Acquired data were processed three-dimensionally using laboratory-made imaging software. Visualization of vessels in the adipose or suprafascial layer was performed using the following steps. First, the surface of the skin was identified automatically using signals from the dermis. Next, color gradation was applied to the images according to the depth from the skin surface. Subdermal venous networks in the most superficial layer were visualized in blue. Finally, vessels in the adipose layer or deeper were visualized independent of the venous networks by semi-automatically cropping out the superficial layer that included the networks (Fig. 1). To study the diagnostic potential of PAT, the correlation between the locations of perforators identified on PAT versus conventional ultrasound (US) was evaluated. To determine the detectability of PAT by depth, the depth of vessels in the stem portion was measured and compared to the depth of the deep fascia measured using US. The orientation of perforators in the stem portion was also evaluated. The branching morphology 
of vessels in the adipose and suprafascial layers was evaluated using three-dimensional observation.

\section{Results}

PAT visualized the branching vessels of perforators in the subcutaneous layer in all subjects. PAT showed

18 perforators and US showed 15 perforators, all of which were visualized by PAT. However, discrepancies

in location of less than $10 \mathrm{~mm}$ were frequently observed. The estimated mean depth of vessels visualized

by PAT was $9 \mathrm{~mm}$; the maximum was $13 \mathrm{~mm}$. Unlike US, PAT does not visualize the soft tissue components.

Comparison of the depth of a vessel visualized by PAT with the depth of the deep fascia visualized by US at

the corresponding site revealed a strong correlation (Pearson's correlation $=0.8$ ). Among the 18 perforators,

nine had an orientation of approximately 30 degrees relative to the horizontal plane and the remaining nine

had an orientation of 30-60 degrees.

Three-dimensional observation of PAT images showed the branching pattern of perforators. Fourteen Type

I and four Type II perforators according to a classification system proposed by Schaverien et al. ${ }^{6}$ were

observed. Figure 2 is a representative image showing a Type II perforator with horizontal branches visualized 
84

\section{Discussion}

This is the first report to show the feasibility of using PAT imaging to visualize the branching morphology of perforators in the subcutaneous layer in the anterolateral thigh. The three-dimensional nature of PAT imaging contributed to identification of the branching morphology of perforators.

Compared with conventional US, PAT visualized vessels to the level of the deep fascia. By contrast, vessels deeper than the fascia were undetectable, suggesting that the deep fascia could be a factor limiting the depth performance of PAT. The lack of anatomical references of PAT imaging is a barrier to its intraoperative use. Cooperation of US with PAT would resolve these issues.

PAT has two other technical limitations. First, there are currently no established methods to distinguish arteries from veins using PAT, thus a triple bundle appearance was common, especially in vessels near the stem portion (Fig. 2). The subdermal venous networks were distinguishable owing to their superficial location and polygonal morphology. ${ }^{7}$ Second, the orientation of a vessel affects its visualization by PAT. This is known as the limited view problem. ${ }^{8}$ More vertically distributed vessels are reportedly less likely to be visualized. Indeed, no vertical vessels were observed in this study, although vessels at an angle of approximately 60 degrees were visualized. This might be the reason why no Type III perforators were found in this study. Although further technical refinements are needed for PAT to establish its clinical utility, we believe that PAT would be a promising new imaging modality for thin ALT flap surgery. 
102 Authors' role/participation in the authorship of the manuscript

103 SS conducted the study and IT performed the experiments. HS developed imaging software. IT, AY and SS

104 analyzed the data. All the authors contributed to writing of the manuscript.

105

106 Acknowledgement

107 This work was funded by the ImPACT Program of the Council for Science, Technology and Innovation

108 (Cabinet Office, Government of Japan). Canon invented the photoacoustic imaging system used in this

109 study. We would like to express our sincere gratitude to all our collaborators: Tomoko Kosaka, Kazuko

110 Kobayashi, Yasufumi Asao, Shuichi Kobayashi and Takayuki Yagi.

111

112 


\section{References}

114

115 1) Kimura N, Satoh K. Consideration of a thin flap as an entity and clinical applications of the thin

116 anterolateral thigh flap. Plast Reconstr Surg. 1996; 97: 985-992.

117 2) Ross GL, Dunn R, Kirkpatrick J, Koshy CE, Alkureishi LW, Bennett N, Soutar DS, Camilleri IG. To thin or

118 not to thin: the use of the anterolateral thigh flap in the reconstruction of intraoral defects. Br J Plast Surg.

119 2003; 56: 409-413.

120 3) Alkureishi LWT, Shaw-Dunn J, Ross GL. Effects of thinning the anterolateral thigh flap on the blood supply

121 to the skin. Br J Plast Surg. 2003; 56: 401-408.

122 4) Wang LV, Hu S. Photoacoustic tomography: in vivo imaging from organelles to organs. Science. 2012; 335(6075):1458-1462.

124 5) Toi M, Asao Y, Matsumoto Y, Sekiguchi H, Yoshikawa A, Takada M, Kataoka M, Endo T, Kawaguchi-Sakita

125 N, Kawashima M, Fakhrejahani E, Kanao S, Yamaga I, Nakayama Y, Tokiwa M, Torii M, Yagi T, Sakurai T,

126 Togashi K, Shiina T. Visualization of tumor-related blood vessels in human breast by photoacoustic imaging

127 system with a hemispherical detector array. Sci Rep. 2017; 7: doi10.1038/srep41970

128 6) Schaverien M, Saint-Cyr M, Arbique G, Hatef D, Brown SA, Rohrich RJ. Three- and four-dimensional 
131 7) Imanishi $\mathrm{N}$, Kishi K, Chang H, Nakajima $\mathrm{H}$, Aiso S. Three-dimensional venous anatomy of the dermis

132 observed using stereography. J Anat. 2008; 212: 669-673.

133 8) Xu Y, Wang LV. Reconstructions in limited-view thermoacoustic tomography. Am Assoc Phys Med. 2004;

$134 \quad 31: 724-733$.

135 


\section{$136 \quad$ Figure legends}

137

$138 \quad$ Fig. 1

139 Anteroposterior photoacoustic tomography images in the all-layer (left) and deep-layer views (right). The

140 color corresponds to the depth from the skin surface. Note that the blue superficial venous networks in the

141 all-layer image are not shown in the deep-layer image; deep branching vessels in red are visualized

142 separately. Scale bar $=20 \mathrm{~mm}$.

143

144 Fig. 2.

145 Photoacoustic tomography projection images showing a Type II branching pattern in a perforator. Insets are

146 lateral views for the regions indicated in the anteroposterior image. In the inset A-B, a perforator bifurcates

147 into vessels coursing horizontally and oblique to the skin surface. In the inset C-D, a branch coursing

148 obliquely from the bifurcation changes its direction, becoming more horizontal in the distal portion. Note the

149 triple bundle appearance of the branches (arrowhead). Scale bars $=10 \mathrm{~mm}$. See also Supplementary video

153 Schematic of the photoacoustic tomography system. 
156 Images show a difference in visual performance between PAT and Doppler ultrasound. Note that the oblique

157 portion of the perforator system in the subcutaneous layer shown by PAT is not visualized by Doppler US

158 (arrowhead).

159

160 Supplemental Digital Content Video 1

161 The video highlights three-dimensional visualization of the branching vessels in Fig. 2 . The color of the

162 vessels corresponds to the depth from the skin. $\mathrm{X}, \mathrm{Y}$, and $\mathrm{Z}$ coordinates indicate the medial, distal, and posterior directions, respectively. 

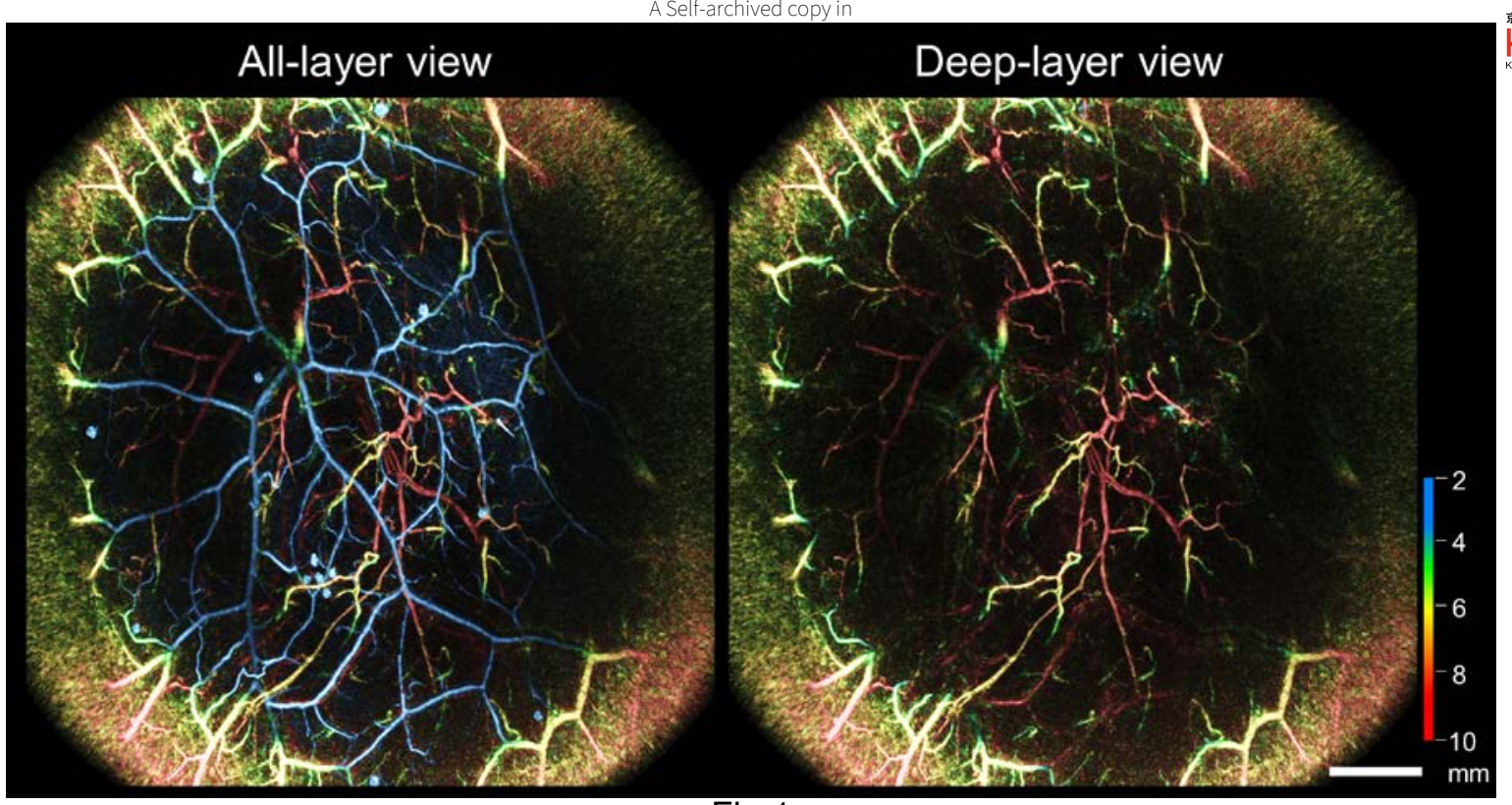

KURENAI 红

Fig.1

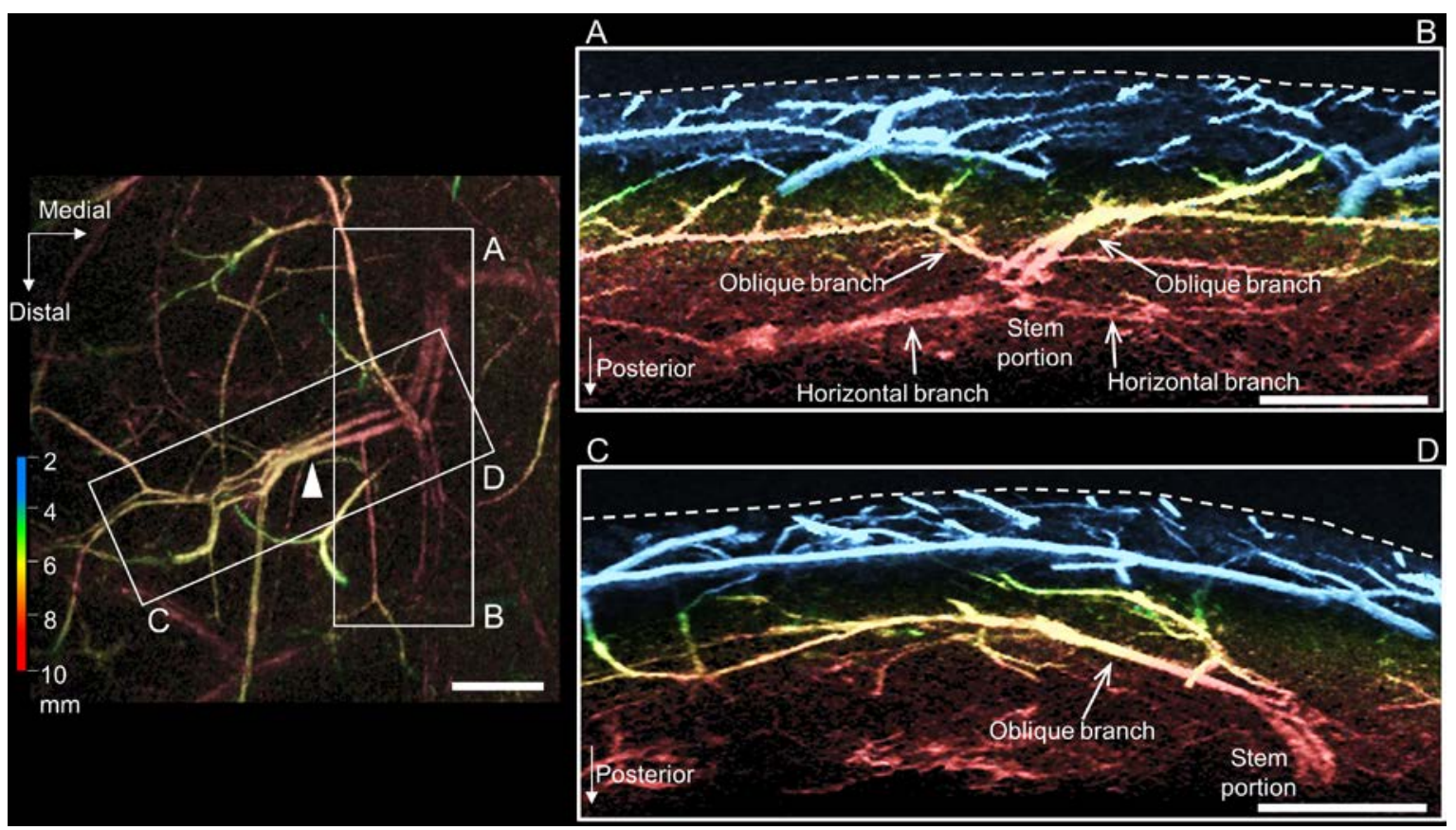

Fig.2

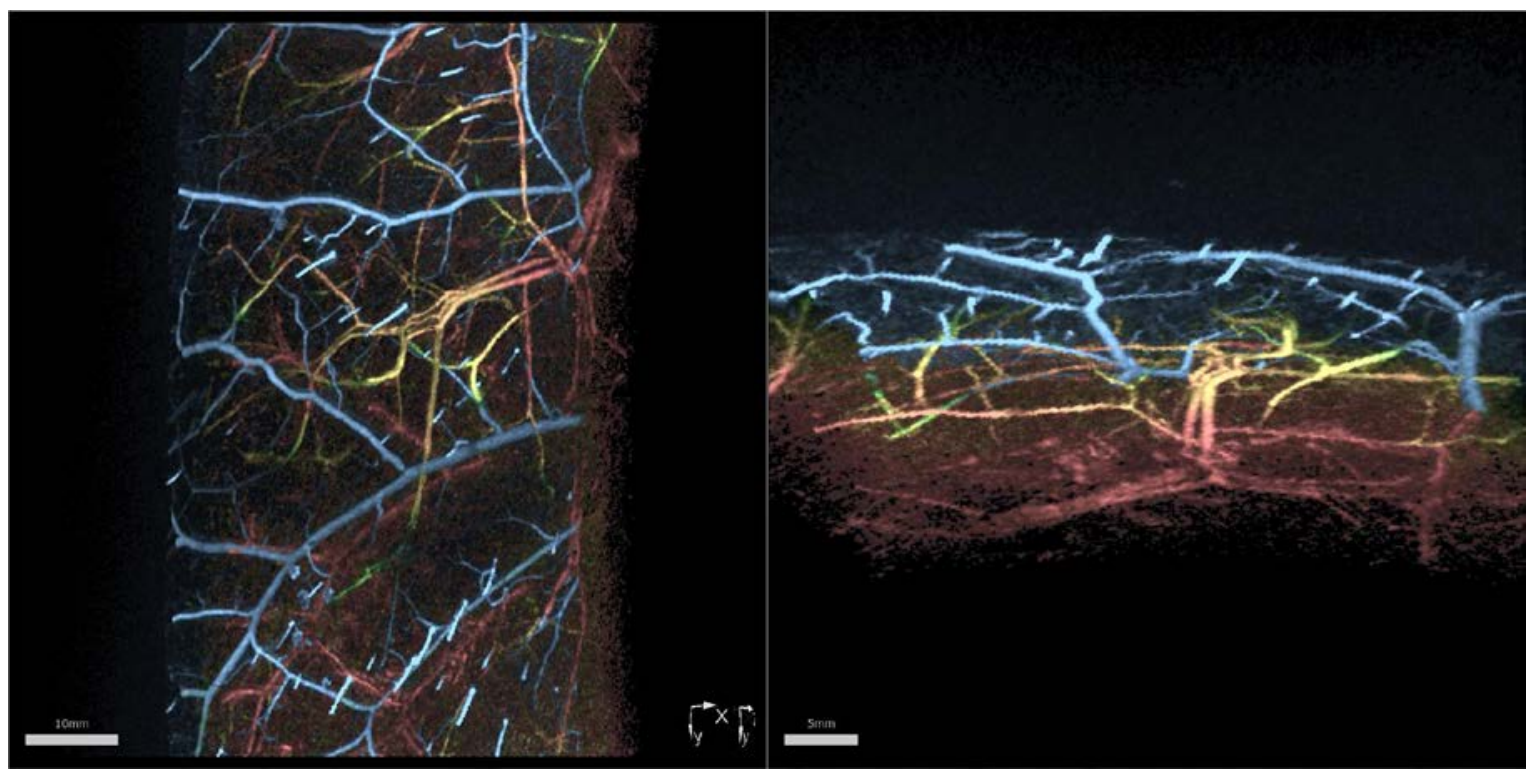




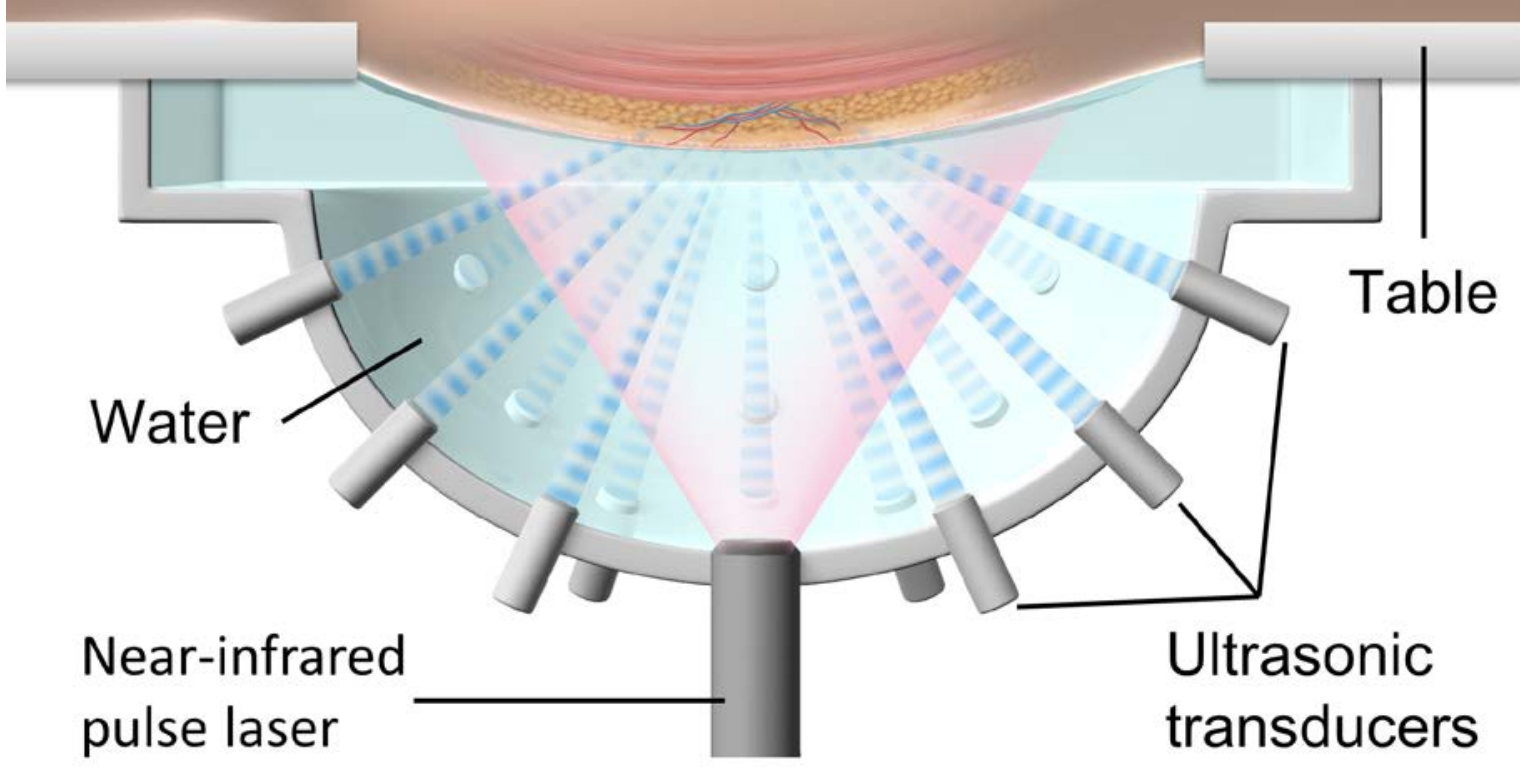

Supplemental Digital Content Figure 1

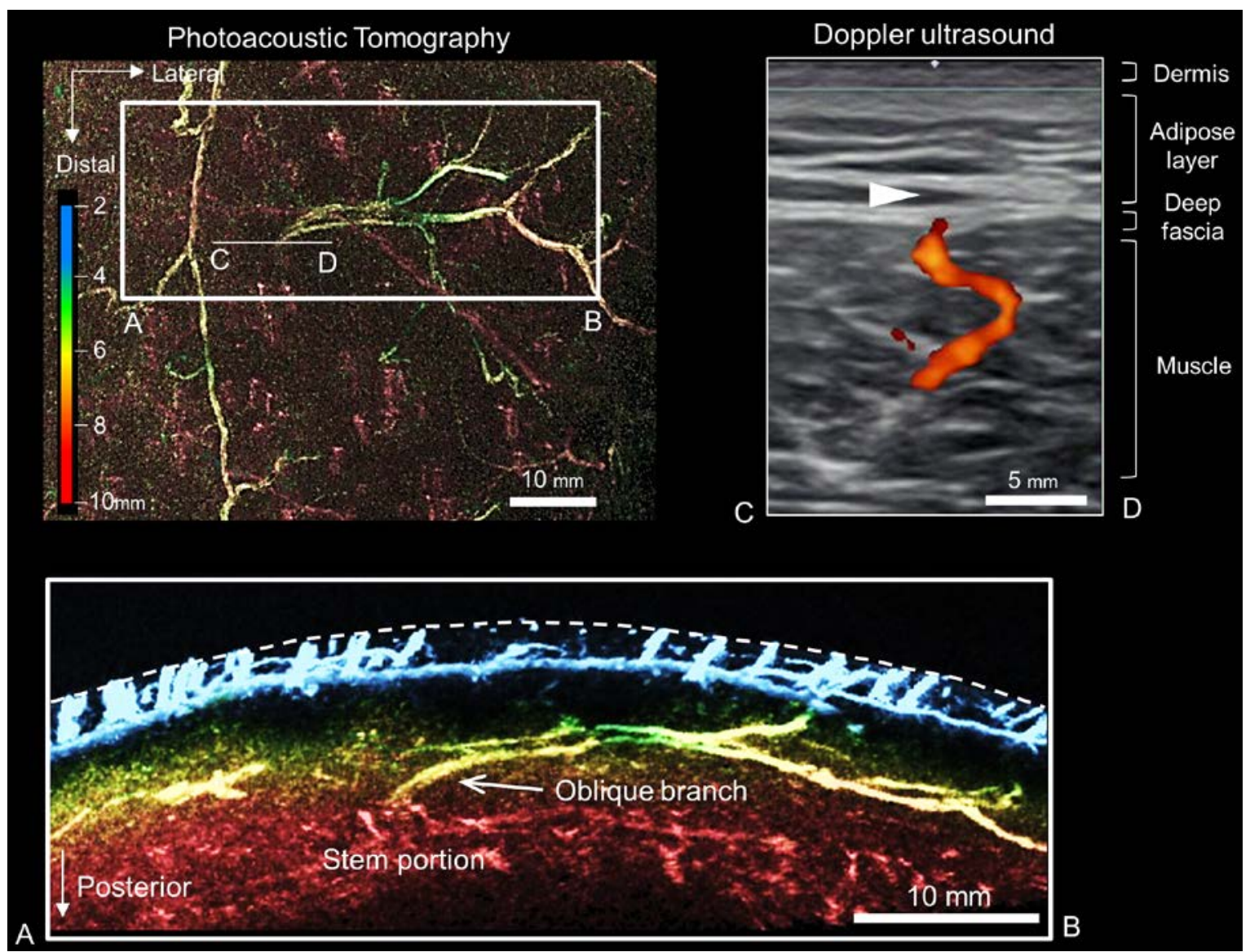

